\title{
An Engineered Calmodulin-Based Allosteric Switch for Peptide Biosensing
}

\section{Citation}

Meister, Glenna E., and Neel S. Joshi. 2013. “An Engineered Calmodulin-Based Allosteric Switch for Peptide Biosensing." ChemBioChem 14 (12): 1460-1467.

\section{Published Version}

10.1002/cbic.201300168;doi:10.1002/cbic.201300168

\section{Permanent link}

http://nrs.harvard.edu/urn-3:HUL.InstRepos:11326226

\section{Terms of Use}

This article was downloaded from Harvard University's DASH repository, and is made available under the terms and conditions applicable to Open Access Policy Articles, as set forth at http:// nrs.harvard.edu/urn-3:HUL.InstRepos:dash.current.terms-of-use\#OAP

\section{Share Your Story}

The Harvard community has made this article openly available.

Please share how this access benefits you. Submit a story.

Accessibility 


\title{
An Engineered Calmodulin-Based Allosteric Switch for Peptide Biosensing
}

\author{
Glenna E. Meister and Neel S. Joshi ${ }^{\left.{ }^{[a, b]}\right]}$
}

This work describes the development of a new platform for allosteric protein engineering that takes advantage of the ability of calmodulin to change conformation upon binding to peptide and protein ligands. The switch we have developed consists of a fusion protein in which calmodulin is genetically inserted into the sequence of TEM1 $\beta$ lactamase. In this approach, calmodulin acts as the input domain, whose ligand-dependent conformational changes control the activity of the $\beta$-lactamase output domain. The new allosteric enzyme exhibits

\section{Introduction}

The development of new technologies for molecular sensing and signal transduction is critical to advancements in fields of synthetic biology, ${ }^{[1]}$ medical diagnostics, ${ }^{[2]}$ and environmental data collection $^{[3]}$ among others. At the most basic level, a molecular sensor should be able to couple two distinct functions: specific molecular recognition (input) and generation of a readily detectable signal (output). Although there exist various methods to accomplish this using advanced analytical techniques such as immunoassays, biology relies heavily on allosteric systems for cellular sensing and signal transduction. Here we broadly define an allosteric system as any protein whose activity is modulated by a binding event at a location distinct from the active site. The study of biological signaling has inspired new methods to engineer allosteric systems, ${ }^{[4]}$ especially those that can sense biomedically relevant targets ${ }^{[5]}$ or interface with established signaling mechanisms in whole cells or organisms. ${ }^{[6]}$

The complexities of protein design have made it difficult to construct allosteric systems de novo, but researchers have circumvented this problem by separating the input and output functions into separate but coupled domains. In this type of approach, an ideal input domain is one that undergoes large but predictable conformational changes upon target binding. The input domain is then fused to an output domain in such a way that the function (i.e. signal generation) of the output domain is conformationally dependent. Despite the abundance of engineered allosteric enzymes and sensors present in the literature ${ }^{[7]}$ few are designed to respond to protein or peptidebased binding targets ${ }^{[8]}$ - rather, most are activated by ions and small molecules. ${ }^{[p]}$ Nevertheless, there is great interest in developing sensors for peptides and proteins because of their central role in cellular signaling. More widespread use of these techniques is hindered by other barriers, such as output signals that are limited to FRET or the need to painstakingly optimize fusion points for the desired output protein, which can require screening of large libraries of randomized fusion points. ${ }^{[9]}$ up to 120 times higher catalytic activity in the activated (peptide bound) state compared to the inactive (no peptide bound) state in vitro. Activation of the enzyme is ligand-dependent - peptides with higher affinities for wild-type calmodulin exhibit increased switch activity. Calmodulin's ability to "turn on" the activity of $\beta$-lactamase makes this a potentially valuable scaffold for the directed evolution of highly specific biosensors for detecting toxins and other clinically relevant biomarkers.

Here we report the rational design and development of a new allosteric switch protein that responds to peptide-based binding targets. Based on the relatively straightforward optimization of the switch compared to related examples, our design could represent a promising platform for modular switch development. The switch we have developed (BLACaM) makes use of the domain insertion technique ${ }^{[7 a]}$ and consists of calmodulin (CaM) inserted into the sequence of TEM1 $\beta$ lactamase (BLA, EC 3.5.2.6). BLACaM is controlled by the conformational changes of the CaM input domain. CaM is a small $(\sim 17 \mathrm{kDa})$ globular protein that exists in three conformational states. In the absence of $\mathrm{Ca}^{2+}$, it adopts a "closed" conformation with its $\mathrm{N}$ - and C-termini spatially separated by only $9 \AA$. In the presence of $\mathrm{Ca}^{2+}, \mathrm{CaM}$ adopts an "open" conformation with the termini separated by $\sim 31 \AA$. In the presence of $\mathrm{Ca}^{2+}$ and a peptide binding target, CaM once again adopts a "closed" conformation (Figure 1a). The target-bound forms of CaM for different peptides are similar but not identical and structural data suggests that the distance between the termini may vary between 13-24 $\AA$ for different targets. Because of its well-characterized conformational dynamics, CaM has been used in molecular sensors such as genetically encodable calcium sensors for decades. However, its ability to bind with high affinity to proteins and peptides has yet to be exploited in allosteric biosensors. Here we show that the new switch, BLACaM, is mostly inactive when $\mathrm{Ca}^{2+}$ is present, and

[a] Dr. N.S. Joshi and Dr. G.E. Meister

School of Engineering and Applied Sciences

Harvard University

29 Oxford St. Cambridge, MA 02143

Fax: 617-432-7828

E-mail:njoshi@seas.harvard.edu

[b] Dr. N.S. Joshi and Dr. G.E. Meister

Wyss Institute for Biologically Inspired Engineering

3 Blackfan Circle, Boston, MA 02115

Supporting information for this article is available on the WWW under http://www.chembiochem.org or from the author. 
becomes activated after binding to a range of target peptides. The switch demonstrates specificity in that it is only activated by peptides that are known to bind to wt-CaM.
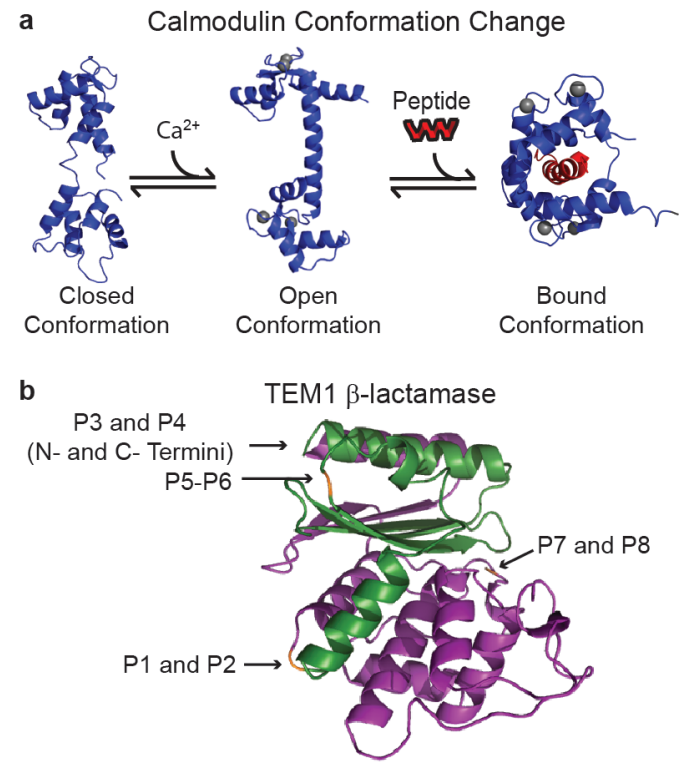

Figure 1. Calmodulin (CaM) and TEM1 $\beta$-lactamase (BLA) structures. a) Structures showing the conformation changes of $\mathrm{CaM}$ in the apo, or closed state (pdb ID: 1CFD), calcium bound, or open state (PDB ID: 1CLL), and bound to calcium and the M13 peptide (PDB ID: 2BBM). b) Structure of the TEM1 $\beta$ lactamase (PDB ID: 1BTL) with the protein complementation fragments BLF1 and BLF2 shown in purple and green, respectively. The insertion points for all switch constructs are indicated with arrows and labelled orange.

\section{Results and Discussion}

\section{Calmodulin - TEM1 $\beta$-lactamase fusions}

Previous attempts at switch engineering using domain insertion required high throughput screening techniques in order to identify a suitable insertion point. ${ }^{[9-10]}$ We sought to avoid the time consuming process of library construction associated with most domain insertion efforts by making use of the known split points of TEM1 $\beta$-lactamase (BLA). Therefore, we synthesized eight BLACaM variants using the BLA split points reported by Galarneau et.al. ${ }^{[11]}$ and Guntas et. al. ${ }^{[9,12]}$ (Figure $1 \mathrm{~b}$ ). We initially constructed genes encoding for a small panel of BLACaM variants (P1-P8, Figure S1). P1 and P2 consisted of the $\mathrm{N}$ terminal fragment of $\mathrm{BLA}(1-194)$ (a.k.a. BLF1) fused to the $\mathrm{N}$ terminus of CaM, and the C-terminal fragment of BLA (196-286) (a.k.a. BLF2) fused to the C-terminus of CaM, while P3 and P4 had the order of the domains reversed. P5/P6 and P7/P8 were analogous to P1/P2 and P3/P4, respectively, except we used insertion points identified by Guntas, et al. in the construction of a maltose binding protein- $\beta$-lactamase switch. ${ }^{[9,12]}$ Our panel included constructs that had no linkers (direct fusion), two very short linkers ( $S$ and $S G$ ), and constructs that used both a short stiff linker (DKS) and a short flexible linker (GSGGG). We rationalized that the Galarneau, et al. split points would be good initial insertion points because the fragments had been preoptimized to: 1) resist spontaneous association in solution, and 2) be individually non-functional but reconstitute an active enzyme when brought in close proximity to one another by fused domains. Additionally, the Guntas, et al. split points, which were identified using a large library $\left(10^{6}\right.$ members $)$ screening process, have already been proven to be suitable insertion sites that allow for modulation of the BLA activity. We reasoned that the open conformation of CaM inserted at these points would be able to separate the two BLA fragments enough to inactivate them. Once CaM adopted a closed conformation in response to ligand binding, the two $\beta$-lactamase fragments would be brought together to activate the enzyme.

The P1-P8 domain insertion variants were initially screened for switching activity in crude cellular lysates. To do this, plasmids encoding for each variant were separately transformed into $E$. coli and overexpression was induced. Following induction, the cells were lysed and the switching properties of the variants were assayed directly in the clarified lysate using a colorimetric indicator (nitrocefin) for BLA activity. The screening process for switching activity was performed by monitoring each variant's BLA activity under sets of conditions known to induce the three different conformational states of wt-CaM (Figure 1a): no calcium or target (1 mM EGTA), calcium only ( $\left.5 \mathrm{mM} \mathrm{Ca}^{2+}\right)$, and calcium plus peptide target ( $5 \mathrm{mM} \mathrm{Ca}^{2+}, 1 \mu \mathrm{M}$ target). The target in this case was the calmodulin binding peptide M13, a 26 amino acid peptide derived from myosin light chain kinase that is known to bind to and induce the "closed" conformation of CaM. ${ }^{[13]}$ Based on the activity assays, the variants were classified into three types: always on (P1 and P3), always off (P2, P5, P7 and P8), and switch (P4 and P6) (Figure 2). The P4 variant exhibited "on" states in both the non-calcium bound and M13 bound states with activity close to baseline ("off") in the presence of calcium while the P6 variant only showed increased activity in the M13 bound state. Notably, both $\mathrm{P} 1$ and $\mathrm{P} 3$ did exhibit higher activity under the EGTA and M13-bound conditions, but also showed significant activity under the calcium only conditions. Furthermore, the range of responses observed for the variants even in this small panel highlights the importance of insertion point and linker flexibility for effective switch construction. We theorized that too much linker flexibility would allow the BLA fragments to associate regardless of the conformational state of the CaM domain, effectively decoupling input from output, while rigid linkers or no linkers may confine the BLA fragments in orientations that do not allow them to re-associate properly. Performing the screen in lysates also enabled us to probe the specificity of the switch. Variants identified as "hits" had to respond specifically to the target peptide in the presence of the complex lysate mixture, which contained proteins, DNA, RNA, lipids, and small molecules.

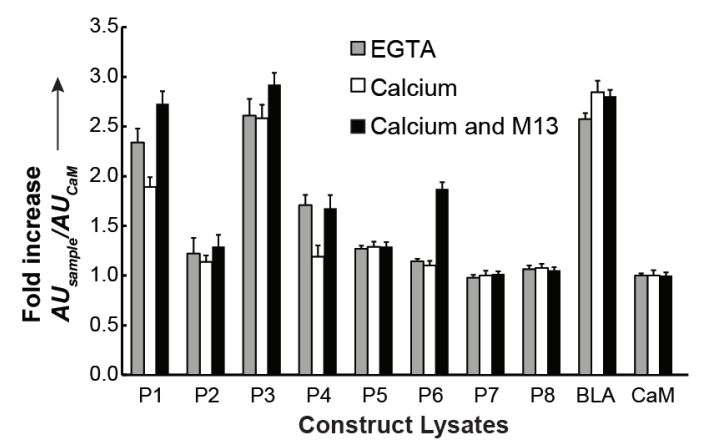

Figure 2. Activity assays for constructs P1-P8 in crude cellular lysates. Total nitrocefin turnover after 7 minutes was monitored at $492 \mathrm{~nm}$ for each construct. Absorbance values are normalized with respect to lysates from cells expressing wt-CaM alone.

\section{BLACaM switch purification and characterization}

Based on the switching activity observed in the lysate assays, the P4 variant (hereafter referred to as BLACaM) was selected for further characterization (Figure 3b). While the P6 fusion also exhibited higher activity when bound to M13 (but not in the presence of EGTA), this switch was found to be highly toxic to $E$. coli cells even in low amounts and was not characterized further. 


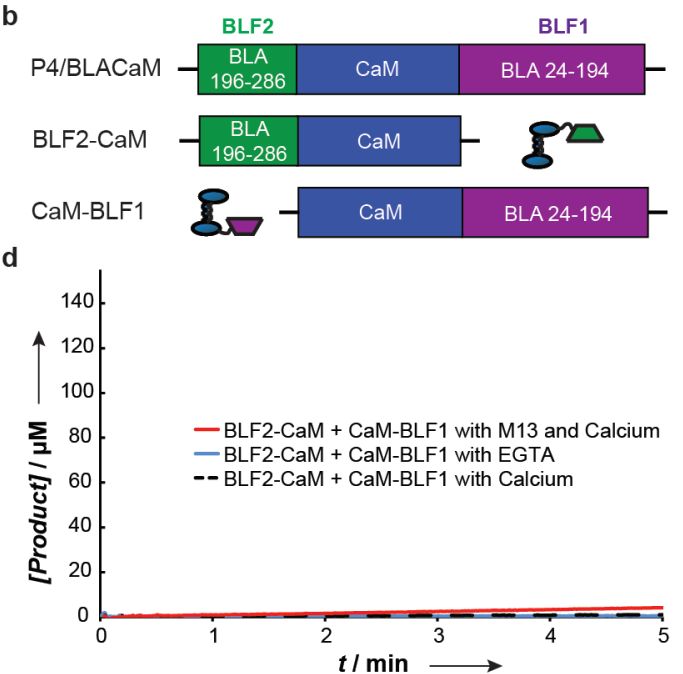

Figure 3. a) Schematic of the BLACaM construct in various conformational states - in the absence of calcium, in the presence of calcium, and bound to calcium and the target peptide M13. b) Schematic of the BLACaM gene construct compared to that of truncation mutants BLF2-CaM and CaM-BLF1. Activity assays were conducted with the CENTA substrate in the presence of $125 \mathrm{nM}$ of either BLACaM (c) or BLF2-CaM and CaM-BLF1 (d) incubated with 5 mM calcium and $500 \mathrm{nM}$ M13 (red), 1 mM EGTA (blue) or 5 mM calcium only (black dashed).

The BLACaM switch also seemed to exhibit a low level of toxicity in $E$. coli cells, however we were able to obtain sufficient amounts of BLACaM using a T7 promoter by expressing at a lower temperature $\left(18^{\circ} \mathrm{C}\right)$ and subsequently purifying using a C-terminal $6 \mathrm{xHis}$ tag. SDS-PAGE characterization of the affinity-purified samples revealed that there was extensive truncation of the protein, possibly resulting from the translation of short fragments from internal start codons. The calm1 gene encoding for the CaM domain originated from humans, and consequently contained several non-optimal codons for $E$. coli that we believe enabled the use of internal start codons and hindered the full translation of BLACaM. Therefore, we altered the gene to include only those codons common in E. coli, allowing for isolation of a BLACaM sample that was $\geq 95 \%$ pure after His-tag affinity purification (Figure S2).

The purified BLACaM protein was then characterized in vitro to determine its switching activity with different analytes. For these experiments, activity was measured by tracking the hydrolysis of a commercially available BLA substrate $\left(\right.$ CENTA $\left.^{\mathrm{TM}}\right){ }^{[14]}$ In order to confirm our hypothesis about the mechanism of switch activation, we ran several control experiments to monitor the activity of BLACaM sub-domains individually. The wt-BLA alone established a baseline for enzyme activity, while wt-CaM alone was not active under any conditions, as expected (Figure S3a). Notably, the activity of pure BLA was decreased slightly in the presence of EGTA. Neither wt-BLA nor wt-CaM was affected by the presence of the target peptide, M13 (Figure S3b). Finally, we conducted activity assays with wt-BLA and wt-CaM together in one pot in the presence of a variety of peptides. In total, the control experiments confirmed three important points: 1) on its own, BLA is largely unaffected by the various conditions expected to induce switching in BLACaM; 2) the CaM domain does not exhibit any ability to hydrolyze the CENTA substrate; 3 ) points 1 and 2 are not affected by noncovalent interactions between BLA and CaM under any of the tested conditions.

With a clear understanding of the behavior of the wild-type proteins, we proceeded to characterize the purified BLACaM switch. As expected from the lysate assays, BLACaM exhibited ligand-dependent activity. In a purely qualitative sense, it was immediately clear that BLACaM was only moderately active in the absence of calcium (EGTA), mostly inactive in the presence of $\mathrm{Ca}^{2+}$ only, and highly active in the presence of $\mathrm{Ca}^{2+}$ plus $\mathrm{M} 13$ (Figure 3a,c). Notably, BLACaM exhibits much higher switching activity when pure compared to when in crude lysate. Also, while the EGTA and $\mathrm{Ca}^{2+}$ plus M13 states appear to be equally active in lysates, they are quite different when pure, suggesting that complex mixtures could have unexpected effects on the switch behavior.

\section{BLACaM activity is due to intramolecular interactions of BLF1 and BLF2}

In a final set of control experiments we wanted to confirm that switch activity was not a result of multimerization of BLACaM during the activity assays. Although the split BLA fragments (BLF1 and BLF2) are designed to have a sufficiently high $\mathrm{K}_{d}$ so as not associate at low concentrations, they can spontaneously refold through intermolecular interactions at sufficiently high concentrations. Therefore, we constructed two truncation mutants of BLACaM (Figure 3b), one with the BLF1 fragment removed (BLF2-CaM) and one with the BLF2 fragment removed (CaMBLF1). In order to probe the potential for intermolecular complementation to contribute to the observed activity of BLACaM, the two truncated mutants were combined at various equimolar concentrations and subjected to activity assays. Some complementation did occur at concentrations above $1 \mu \mathrm{M}$ (Figure S4c), but no significant substrate turnover was observed at concentrations that were used for the BLACaM activity assays (125 nM) (Figure 3d).

Based on these experiments, it is clear that multimerization was not a factor in the switching activity experiments. However, it should be noted that the switch needed to be "primed" by calcium-induced inactivation before rigorous switching activity experiments could take place. BLACaM required $50+$ minutes of incubation with $\mathrm{Ca}^{2+}$ at room temperature to reach its lowest activity levels (Figure S5a), possibly due to residual intramolecular fragment complementation. In contrast, after being in the "off" state, the switch could be reactivated again in less than 5 minutes upon addition of the target peptide M13 (Figure $\mathrm{S} 5 \mathrm{~b})$, suggesting that fragment association from an unfolded state occurs much more rapidly than dissociation of the folded fragments. It is known that certain assisted protein complementation fragments are stabilized in their fully folded state, which could explain the longer times required for inactivation. ${ }^{[15]}$ Notably, the time required for switch inactivation was dramatically reduced by increases in temperature. BLACaM that was assayed following a 10 minute incubation with only $\mathrm{Ca}^{2+}$ at $37^{\circ} \mathrm{C}$ followed by re-equilibration at $25^{\circ} \mathrm{C}$ was inactive, but remained active when incubated with M13 (Figure S6a). Activity assays performed at various temperatures confirmed that BLACaM retains most of its activity up to $30^{\circ} \mathrm{C}$ in the EGTA or calcium plus peptide states. However, at $37^{\circ} \mathrm{C}$, the switch loses 


\begin{tabular}{|c|c|c|c|c|}
\hline & Peptide Sequence & $\begin{array}{l}\text { Switching Activity } \\
\text { (SA) })^{\mathrm{a}}\end{array}$ & $\begin{array}{l}\text { \# of Binding } \\
\text { Sites }\end{array}$ & $\begin{array}{l}\mathrm{K}_{\mathrm{d}} \text { with } \\
\text { wt-CaM }\end{array}$ \\
\hline Calcium only & N/A & $1.0 \pm 0.1$ & 4 & $\mathrm{~N} / \mathrm{A}$ \\
\hline EGTA & N/A & $29 \pm 2$ & - & $\mathrm{N} / \mathrm{A}$ \\
\hline M13 (sm-MLCK fragment) & KRRWKKNFIAVSAANRFKKISSSGAL & $110 \pm 12$ & 1 & $0.13 \mathrm{nM}^{b}$ \\
\hline Mastoparan & INLKALAALAKKIL & $120 \pm 11$ & 1 & $0.3 \mathrm{nM}^{b}$ \\
\hline Melittin & GIGAVLKVLTTGLPALISWIKRKRQQ & $100 \pm 11$ & 1 & $3 \mathrm{nM}^{b}$ \\
\hline$\delta$-toxin & MAQDIISTIGDLVKWIIDTVNKFTKK & $16 \pm 2$ & 1 & $c$ \\
\hline Neuropeptide Y (NPY) & YPSKPDNPGEDAPAEDMARYYSALRHYINLITRQRY & $5.2 \pm 0.7$ & 1 & c \\
\hline $\begin{array}{l}\text { Luteinizing Hormone- Releasing } \\
\text { Hormone (LH-RH) }\end{array}$ & pEHWSKGLRPG & $0.97 \pm 0.13$ & 1 & $--^{d}$ \\
\hline Angiotensin & DRVYIHPFHL & $1.1 \pm 0.1$ & 1 & $--^{d}$ \\
\hline Trifluoperazine (TFP) & N/A & $2.2 \pm 0.3$ & $4+$ & $--^{e}$ \\
\hline
\end{tabular}

[a] A description of the statistical analysis is available in the Supporting Information. [b]Published dissociation constants for CaM binding to M13, ${ }^{[16]}$ mastoparan, ${ }^{[17]}$ and melittin. ${ }^{[18]}[\mathrm{c}]$ While binding was confirmed for NPY ${ }^{[19]}$ and $\delta$-toxin ${ }^{[20]}$ accurate $\mathrm{K}_{d}$ determinations were not done. [d] There is no known interaction between CaM and these peptides. [e] Multiple TFP molecules bind cooperatively to CaM. ${ }^{[21]}$

all activity in the absence of calcium, but remains marginally active when bound to the target peptide (Figure S6b,d). This suggests that the peptide-bound conformation of CaM helps stabilize the BLA fragments, which may contribute to the difference in observed activity between the EGTA and the $\mathrm{Ca}^{2+}$ plus M13 conditions (Figure $3 \mathrm{c}$ ).

\section{Switching Activity (SA) of BLACaM switch}

CaM is known to bind to a variety of peptide and protein targets, making it a potentially useful starting point for the design of input domains with greater affinity and specificity for many target analytes. Accordingly, we screened BLACaM switching activity in response to a panel of targets that spanned a range of binding affinities for wt-CaM. We defined a parameter SA as the ratio of the initial rates of substrate hydrolysis in the presence of calcium plus the target (or EGTA) compared to the mostly inactive calcium bound state: $\mathrm{SA}=\mathrm{V}_{0}{ }^{\text {peptide }} / V_{0}{ }^{\text {Calcium }}$.

Table 1 displays the SA values for BLACaM in response to a 4-fold molar excess of each peptide target. Peptide targets that are known to bind to wt-CaM with high affinities (M13, ${ }^{[16]}$ mastoparan, ${ }^{[17]}$ melittin $^{[18]}$ ) exhibited SA values ranging from 100120. Targets with moderate affinity for wt-CaM (NPY, ${ }^{[19]} \delta$ toxin $^{[20]}$ ) exhibited lower SA values. Finally, targets with no known affinity for CaM (LH-RH, angiotensin) had SA values close to 1 . Additionally, we evaluated the effect of the small molecule trifluoperazine (TFP), which is known to cause a calciumdependent conformational change in $\mathrm{CaM}^{[21]}$ on the BLACaM switch. However, only a slight ( 2-fold) increase in activity was observed. This data set supports the claim that the mechanism of switch activation is through ligand-induced conformational changes of the CaM-based input domain. Among the target ligands that activate the allosteric switch are peptides that may be particularly useful as clinically relevant biomarkers. In particular, neuropeptide $\mathrm{Y}$ (NPY) is a neurotransmitter hormone that has been proposed as biomarker to monitor conditions ranging from anorexia ${ }^{[22]}$ to post traumatic stress disorder (PTSD). ${ }^{[23]}$ Similarly, $\delta$-toxin is an endotoxin that is routinely used to identify virulent strains of $S$. aureus, ${ }^{[24]}$ an opportunistic pathogen responsible for many infections.

\section{Activity of the BLACaM switch is dose dependent}

In order to determine the sensitivity of the switch towards a target ligand, we monitored substrate hydrolysis rates for BLACaM at various concentrations of M13. A plot of initial rates versus $M 13$ concentration reveals that the peptide can be detected at tens of nanomolar concentrations and is dose dependent up to $150 \mathrm{nM}$ (Figure 4). There was $\sim 10-20 \%$ decrease in initial rates for $\mathrm{M} 13$ concentrations above $175 \mathrm{nM}$, hindering the effectiveness of traditional Michaelis-Menten-type kinetic models to fit the data (data not shown). We attribute this rate decrease to aggregation of $\mathrm{M} 13$, which is a known problem in CaM binding assays because many canonical CaM binders are hydrophobic peptides prone to aggregation in aqueous solutions. ${ }^{[20,25]}$

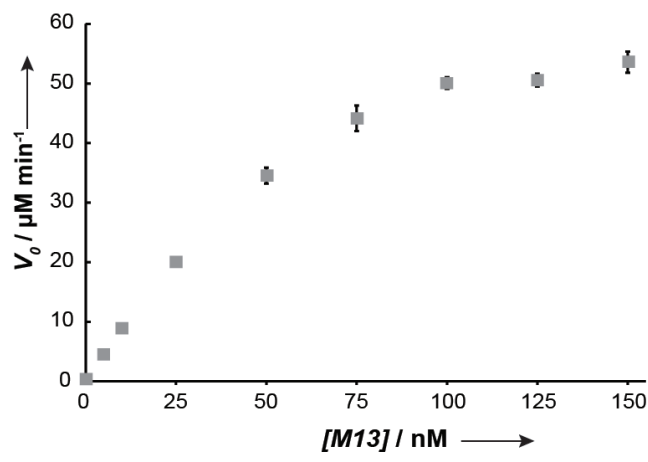

Figure 4. Initial rates of substrate hydrolysis as a function of M13 concentration for BLACaM in the presence of $\mathrm{Ca}^{2+}$.

\section{Conclusion}

We have developed a new allosteric enzyme by inserting CaM into a previously established split point for BLA. In contrast to previous attempts to create allosteric enzymes by domain insertion, the initial identification of this switch structure was accomplished without the need to synthesize and analyze large libraries of mutants. Furthermore, the switch is activated to various degrees (up to 120 -fold) by several peptides and one small molecule target with reported affinity for wt-CaM. This magnitude of ligand-dependent activation is on par with the some of the highest values reported for both natural and engineered allosteric enzymes. ${ }^{[9]}$ This represents the first example of a calmodulin-based allosteric biosensor designed to respond to peptide targets. We anticipate that the BLACaM architecture will be a fruitful starting point for the rational design or directed evolution of new highly specific and sensitive biosensors for use inside cells and in vitro.

\section{Experimental Section}

General: All reagents were purchased from Sigma and all enzymes from New England Biolabs unless otherwise noted. Molecular cloning techniques and protein analysis such as PCR and electrophoresis were performed as described in Sambrook and Russell. ${ }^{[26]}$ 
Gene Construction of Calmodulin and $\beta$-lactamase Fusions: Fusions of calmodulin and $\beta$-lactamase were created by overlap extension PCR using the Phusion ${ }^{\circledR}$ DNA Polymerase (NEB) and primers from either Eurofins MWG Operon or Bioneer Corp (see SI Table 1). All restriction enzymes and T4 DNA ligase were purchased from New England Biolabs. Cloning was performed in the Turbo electrocompetent cell line (New England Biolabs). The calmodulin gene (human variant calm1) was obtained from Origene Technologies Inc. and TEM1 $\beta$-lactamase gene (b/a) was cloned from the pDIMN2 vector. ${ }^{[27]}$

We created constructs fusing calmodulin (CaM) with TEM1 $\beta$-lactamase at four different fusion points: 1) the protein complementation assay (PCA) split points at BLA 194 and 196, 2) the BLA N- and C-termini using the (PCA) fragments, ${ }^{[28]} 3$ ) fusion points of the RG13, ${ }^{[12]}$ and 4) MBP317-347 ${ }^{9]}$ maltose binding protein(MBP)-BLA allosteric switches discovered by Guntas. PCR fragments of all BLA fragments were made using the TEM1 $\beta$ lactamase gene from the pDIMN2 vector as a template and using primers to either fuse fragments directly to CaM or with short linkers DKS and GSGGG. The BLA fragments utilized for fusions P1-P4 were the PCA fragments BLF1 (BLA 24-194) and BLF2 (BLA196-286), for fusions P5-P6 were fragments from RG13 (BLA 24-226 and 227-286), and for P7 and P8 from MBP317-347 (BLA 24-170 and BLA 170-286). All fragments were created by $\mathrm{PCR}$ using the following primers in Table $\mathrm{S} 1$. The human calmodulin gene, calm1, was amplified by PCR for fusion to BLA either directly or with described linkers. For ease of cloning, the later fusions (P5-P8) used either the DKS and GSGGG linkers or much shorter linkers of only $S$ and $S G$ to insert restriction sites of Pstl and BspEl sites respectively. The various fragments of bla and calm1 DNA were then mixed together in equimolar amounts for an overlap extension PCR step and amplified with either BLA sig (Bglll)-for and BLA-rev (P1 and P2), BLF2-for and BLF1-rev ( $\mathrm{P} 3$ and $\mathrm{P} 4)$, or BLA24(BamHI)-for and BLA-rev (P5-P8) primers. Assembled fragments (see Figure S1) were then gel purified (Qiagen Gel Purification Kit) and digested with either Bglll and Spel or BamHI and Spel for cloning into pDIMC8 vectors digested with $\mathrm{BamHI}$ and Spel. Fragments were then ligated into the digested pDIMC8 vector with T4 DNA ligase, transformed into Turbo electrocompetent cells for cloning, and then into DH5a cells for expression. Control vectors containing the wt-bla and wtcalm1 genes were also created by amplifying the wild-type genes using the BLA sig (BgllI)-for/BLA-rev and Calml(BamHI)for/Calml(Spel)-rev primers, digesting and inserting into the pDIMC8 vector.

Construction of BLACaM gene constructs and controls: For purification of the BLACaM fusion, an optimized version of the gene for expression in E. coli was designed using Gene Designer software from DNA 2.0 and synthesized by Biomatik. This gene version was cloned into the pET30a vector (EMD Millipore) appending a $6 \mathrm{x}$ His tag. All controls for expression were also cloned into the pET30a vector appending the $6 \mathrm{x}$ His tag and under control of a T7 promoter. CaM, BLA2-CaM, and CaM-BLA1 controls were created by cloning sections of the optimized BLACaM gene using primer pairs BLA2opt-for/CaMopt(Xhol)-rev and CaMopt-for/BLA1opt(Xhol)-rev respectively. A wt-bla gene was also inserted into the $\mathrm{pET} 30 \mathrm{a}$ expression vector appending a $6 \mathrm{xHis}$ tag for purification.

BLACaM expression and purification: An optimized version of BLACaM for expression in E. coli in the pET30a vector (EMD Millapore) attached to a 6xHis tag and transformed into Shuffle T7 LysY cells (New England Biolabs). Cells were grown in LB media supplemented with $50 \mu \mathrm{g} / \mathrm{ml}$ kanamycin until an OD of 0.6 0.8 at $30^{\circ} \mathrm{C}$. Protein expression was then induced with $0.4 \mathrm{mM}$ IPTG and expressed at $18^{\circ} \mathrm{C}$ for $14-16 \mathrm{hrs}$. Cells were resuspended in lysis buffer $(50 \mathrm{mM}$ Tris-CL, pH 7.4 with $300 \mathrm{mM}$ $\mathrm{NaCl}, 20 \mathrm{mM}$ imidazole and cOmplete EDTA-free protease inhibitor cocktail (Roche)), lysed by sonication, and spun down to clarify lysate. Before application to the gravity column with $\mathrm{Ni}$ NTA agarose (Qiagen), Tween-20 was added to the lysate to a $1 \%$ concentration. Lysate was applied to the column equalibriated with 5 column volumes of $50 \mathrm{mM}$ Tris-CL, pH 7.4 with $300 \mathrm{mM}$
$\mathrm{NaCl}, 20 \mathrm{mM}$ imidazole and $1 \%$ tween and washed with 5 column volumes of the same buffer after lysate application. Column was then washed with an additional 5 column volumes of buffer without tween but containing $50 \mathrm{mM}$ imidazole, and then eluted with 3 column volumes of buffer containing $150 \mathrm{mM}$ imidazole.

BLF2-CaM and CaM-BLF1 controls expression and purification: pET30a BLF2-CaM vector was transformed into BL21 (DE3) cells (Invitrogen) while the pET30a CaM-BLF1 vector was transformed into SHuffle T7 lys $\mathrm{Y}$ cells (New England Biolabs) for expression. Controls were expressed and purified using the same protocol as for BLACaM except that the BL21(DE3) cells were originally grown at $37^{\circ} \mathrm{C}$ instead of the $30^{\circ} \mathrm{C}$ at which SHuffle $\mathrm{T} 7$ lys $\mathrm{Y}$ cells are grown.

Calmodulin (CaM) expression and purification: The pET30a with optimized CaM was transformed into BL21 (DE3) cells and expressed using same procedure as BLACaM. Purification of CaM was performed essentially as described by Gopalakrishna and Anderson. ${ }^{[29]}$

TEM1 $\beta$-lactamase (BLA) expression and purification: $p E T 30 a$ BLA was transformed into BL21 (DE3) cells for expression. Cells were grown to an OD of 0.6-0.8 and induced with 0.4 mM IPTG. BLA was expressed at $37^{\circ} \mathrm{C}$ for 4 hours. Protein was purified on a Ni-NTA agarose column in protocol similar to BLACaM, however no Tween-20 was used in any buffers.

Lysate Screening Assays: DH5a cells were transformed with pDIMC8 plasmids with various CaM-BLA fusion constructs as well as a plasmid containing wt-bla and wt-calm1 were grown in $50 \mathrm{ml}$ LB media supplemented with $50 \mu \mathrm{g} / \mathrm{ml}$ chloramphenicol. Cells were grown at $37^{\circ} \mathrm{C}$ to an $\mathrm{OD}$ at $600 \mathrm{~nm}$ to approximately 0.5-0.6 and induced with $1 \mathrm{mM}$ IPTG and protein was expressed for an additional 4 hours. Cells wre then spun down and frozen overnight. Cells were then resuspended in $2.5 \mathrm{ml}$ lysis buffer (50 $\mathrm{mM}$ Tris-CL, pH 7.5 and cOmplete EDTA-free protease inhibitor cocktail (Roche)) and cells lysed by freeze/thaw method and clarified by centrifugation. After clarification, the lysates' total protein concentrations were determined using the Bio-Rad Quick Start Bradford Protein Assay kit. Samples were diluted to a total protein concentration of $\sim 725 \mu \mathrm{g} / \mathrm{ml}$ and $25 \mu \mathrm{l}$ was mixed with $100 \mu \mathrm{l}$ of $50 \mathrm{mM}$ Tris-Cl buffer with mixtures containing final concentrations of either $1 \mathrm{mM} \mathrm{EGTA}, 5 \mathrm{mM} \mathrm{CaCl}_{2}$, or $5 \mathrm{mM} \mathrm{CaCl}$ ${ }_{2}$ plus $1 \mu \mathrm{M} \mathrm{M} 13$ peptide ( $\mathrm{N}=3$ for each condition). Mixtures were then incubated for $1 \mathrm{hr}$ at room temperature. After incubation 100 $\mu \mathrm{l}$ of lysate mixture was transferred to another microassay plate with $5 \mu \mathrm{l}$ of $4.25 \mathrm{mM}$ nitrocefin substrate (EMD Millipore) in 50 $\mathrm{mM}$ Tris-CL and mixed. Absorbances at $492 \mathrm{~nm}$ were read $\sim 25$ min after mixing on a Biotek Synergy $\mathrm{H} 1 \mathrm{Hybrid}$ plate reader.

BLACaM Activity Assays: Assays were done in the presence of $20 \mathrm{mM}$ HEPES with either $5 \mathrm{mM} \mathrm{CaCl} 2,1 \mathrm{mM}$ EGTA, or $5 \mathrm{mM}$ $\mathrm{CaCl}_{2}$ and peptide at $500 \mathrm{nM}$ or trifluoperazine (TFP) at $1 \mu \mathrm{M}$ unless otherwise noted. Peptides were obtained from Anaspec in $\geq 95 \%$ purity and the small molecule TFP was obtained from Sigma. Buffer was mixed with $125 \mathrm{nM}$ protein and incubated for 50 minutes at room temperature. Two $\mathrm{ml}$ of protein mixtures were then transferred to a cuvette and incubated in the Cary UV-Vis spectrophotometer (Agilent Technologies) with stirring for $10 \mathrm{~min}$ to equilibrate to $25^{\circ} \mathrm{C}$ or other assay temperature. At time zero, $150 \mu \mathrm{M}$ CENTA ${ }^{\mathrm{TM}}$ substrate (EMD Millipore) which was added to start the assay. Product concentration was determined by monitoring absorbance at $405 \mathrm{~nm}$ and using the CENTA ${ }^{\mathrm{TM}}$ extinction coefficient: $\Delta \varepsilon=6400 \mathrm{M}^{-1} \mathrm{~cm}^{-1}$ to calculate product concentrations. To determine switching activity, assays were performed for 3 different batches of purified BLACaM with multiple runs ( $\mathrm{n}=3$ to 8 ) for each peptide target and buffer condition. $V_{0}$ data was calculated for each run using the Cary WinUV Software and batch switching activity $\left(V_{0}^{\text {condition }} / V_{0}{ }^{\text {calcium }}\right)$ and standard deviation of the mean (SDM) determined. Reported SA values were then calculated using a weighted average of the three batch's values. Dose dependent $V_{0}$ data using different concentrations of M13 peptide were averaged from multiple runs 
$(n=4)$. Alternatively, assays were also performed in 96 well microassay plates using the same concentrations and general procedures but with only $100 \mu \mathrm{l}$ total volume and absorbances read using the Biotek Synergy $\mathrm{H} 1$ Hybrid plate reader.

\section{Acknowledgements}

The authors would like to thank the National Science Foundation (CBET - 1158784) for supporting this work.

\section{Keywords: (allostery · biosensors · calmodulin · domain} insertion · protein engineering)

[1] a) M. V. Golynskiy, M. S. Koay, J. L. Vinkenborg, M. Merkx, ChemBioChem 2011, 12, 353-361; b) W. C. Ruder, T. Lu, J. J. Collins, Science (New York, NY) 2011, 333, 1248-1252.

[2] a) J. H. Lee, M. V. Yigit, D. Mazumdar, Y. Lu, Advanced Drug Delivery Reviews 2010, 62, 592-605; b) J. Lueke, W. A. Moussa, Sensors 2011, 11, 1433-1460.

[3] a) D. Ivnitski, I. Abdel-Hamid, P. Atanasov, E. Wilkins, Biosensors and Bioelectronics 1999, 14, 599-624; b) S. Liu, Z. Zheng, X. Li, Analytical and Bioanalytical Chemistry 2012, 405, 63-90.

[4] F. Fan, B. F. Binkowski, B. L. Butler, P. F. Stecha, M. K. Lewis, K. V. Wood, ACS chemical biology 2008, 3, 346-351.

[5] a) O. Laczka, R. Ferraz, N. Ferrer-Miralles, A. Villaverde, F. Muñoz, F. Campo, Analytica chimica acta 2009, 641, 1-6; b) S. H. NajafiShoushtari, M. Famulok, Blood Cells, Molecules, and Diseases 2007, 38, 19-24.

[6] B. J. Yeh, R. J. Rutigliano, A. Deb, D. Bar-Sagi, W. A. Lim, Nature 2007, 447, 596-600.

[7] a) M. Ostermeier, Protein Eng Des Sel 2005, 18, 359-364; b) A Vallee-Belisle, K. W. Plaxco, Curr Opin Struct Biol 2010, 20, 518526.

[8] J. Huang, S. Koide, ACS chemical biology 2010, 5, 273-277.

[9] G. Guntas, T. J. Mansell, J. R. Kim, M. Ostermeier, Proc Natl Acad Sci U S A 2005, 102, 11224-11229.

10] G. Guntas, M. Kanwar, M. Ostermeier, PLoS One 2012, 7, e35998.

[11] A. Galarneau, M. Primeau, L. Trudeau, S. Michnick, Nature Biotechnology 2002, 20, 619-622.

[12] G. Guntas, S. F. Mitchell, M. Ostermeier, Chem Biol 2004, 11, 1483 1487.
D. Blumenthal, K. Takio, A. Edelman, H. Charbonneau, K. Titani, K. Walsh, E. Krebs, Proceedings of the National Academy of Sciences $1985,82,3187$.

[14] C. Bebrone, C. Moali, F. Mahy, S. Rival, J. D. Docquier, G. M. Rossolini, J. Fastrez, R. F. Pratt, J. M. Frere, M. Galleni, Antimicrob Agents Chemother 2001, 45, 1868-1871.

[15] M. Morell, S. Ventura, F. X. Aviles, FEBS letters 2009, 583, 1684 1691.

[16] D. F. Green, A. T. Dennis, P. S. Fam, B. Tidor, A. Jasanoff, Biochemistry 2006, 45, 12547-12559.

[17] D. A. Malencik, S. R. Anderson, Biochem Biophys Res Commun 1983, 114, 50-56.

[18] Y. Maulet, J. A. Cox, Biochemistry 1983, 22, 5680-5686

[19] K. Kitamura, K. Kangawa, K. Tanaka, H. Matsuo, Biochemical and Biophysical Research Communications 1990, 169, 1164-1171.

[20] L. Garone, J. E. Fitton, R. F. Steiner, Biophys Chem 1988, 31, 231245.

[21] M. Vandonselaar, R. A. Hickie, J. W. Quail, L. T. Delbaere, Nature structural biology 1994, 1, 795-801.

[22] E. Yulyaningsih, L. Zhang, H. Herzog, A. Sainsbury, British Journal of Pharmacology 2011, 163, 1170-1202.

[23] D. Hirsch, Z. Zukowska, Cellular and Molecular Neurobiology 2012, 32, 645-659.

[24] R. Wang, K. R. Braughton, D. Kretschmer, T. H. Bach, S. Y. Queck M. Li, A. D. Kennedy, D. W. Dorward, S. J. Klebanoff, A. Peschel, F. R. DeLeo, M. Otto, Nature medicine 2007, 13, 1510-1514.

[25] R. Stevens-Truss, M. A. Marletta, Biochemistry 1995, 34, 1563815645.

[26] J. Sambrook, D. W. Russell, Molecular Cloning: A Laboratory Manual, 3rd ed., Cold Spring Harbor Laboratory Press, Cold Spring Harbor, NY, 2001

[27] D. E. Paschon, Z. S. Patel, M. Ostermeier, J Mol Biol 2005, 353, 2637

[28] A. Galarneau, M. Primeau, L. E. Trudeau, S. W. Michnick, Nat Biotechnol 2002, 20, 619-622.

[29] R. Gopalakrishna, W. B. Anderson, Biochemical and Biophysical Research Communications 1982, 104, 830-836.

Received: ((will be filled in by the editorial staff))

Published online: ((will be filled in by the editorial staff)) 
Entry for the Table of Contents (Please choose one layout)

Layout 1:

\section{FULL PAPERS}

((Text for Table of Contents))

((Author(s), Corresponding Author(s)*)

Page No. - Page No.

((Title))

((Insert TOC Graphic here))

Layout 2:

\section{FULL PAPERS}

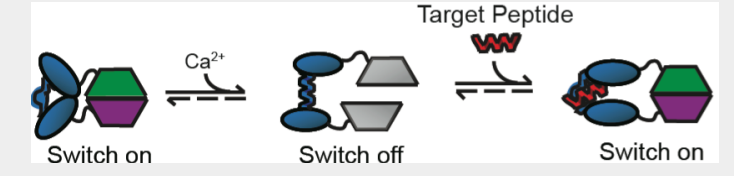

A calmodulin based switch detects peptides by switching on $\beta$-lactamase activity in the presence of both calcium and the peptide.
Glenna E. Meister and Neel S. Joshi*

Page No. - Page No.

Engineered Calmodulin-Based Allosteric Switch for Peptide Biosensing 


\title{
Engineered Calmodulin-Based Allosteric Switch for Peptide Biosensing
}

\author{
Glenna E. Meister and Neel S. Joshi ${ }^{a, b}$
}

${ }^{[a]}$ Harvard University, School of Engineering and Applied Sciences

${ }^{[b]}$ Wyss Institute for Biologically Inspired Engineering

njoshi@seas.harvard.edu

SI Table 1: Primers used in cloning. Primers were purchased from either Eurofins MWG Operon or Bioneer Corp.

\begin{tabular}{|c|c|}
\hline Primer Name & Primer Sequence (5'-3') \\
\hline \multicolumn{2}{|l|}{ P1 and P2 Primers } \\
\hline BLA sig (BgllI)-for & GCACCCAGATCTCATATGAGTATTCAACATTTCCGTGTCGCCC \\
\hline BLA nosig(BgllI)-for & GCACCCAGATCTCATATGCACCCAGAAACGCTGGTGAAAG \\
\hline BLA-rev & CCTACGACTAGTCTATTACCAATGCTTAATCAGTGAGGCACCTATCTC \\
\hline BLF1-DKS-rev & GGTCAGCTGATCTGCAGATTTATCGCCAGTTAATAGTTTGCGCAACGTTGTTGC \\
\hline Calm1-DKS-for & GGCGATAAATCTGCAGATCAGCTGACCGAAGAACAGATTGC \\
\hline Calm1-GSGGG-rev & CCGCCTCCGGACCCTTTTGCAGTCATCATCTGTACGAATTCTTCATAGTTGACTTGTCC \\
\hline BLF2-GSGGG- for & GCAAAAGGGTCCGGAGGCGGTCTACTTACTCTAGCTTCCCGGCAACAATTAATAGACTGG \\
\hline BLF1-Calm1-rev & GCTGATCTGCGCCAGTTAATAGTTTGCGCAACGTTGTTGC \\
\hline Calm1-BLF1-for & GCAAACTATTAACTGGCGCAGATCAGCTGACCGAAGAACAGATTGC \\
\hline Calm1-BLF2-rev & GCTAGAGTAAGTAGTTTTGCAGTCATCATCTGTACGAATTCTTCATAGTTGACTTGTCC \\
\hline BLF2-Calm1-for & CGTACAGATGATGACTGCAAAACTACTTACTCTAGCTTCCCGGCAACAATTAATAGACTGG \\
\hline BLA(Spel)-rev & CCACGAAGCTTACTAGTCTATTACCAATGCTTAATCAGTGAGGCACCTATCTCAGC \\
\hline BLA sig (BamHI)-for & CGGTGGGATCCATATGAGTATTCAACATTTCCGTGTCGCCC \\
\hline \multicolumn{2}{|l|}{ P3 and P4 Primers } \\
\hline BLF2-for & CGGTGGGATCCATATGCTACTTACTCTAGCTTCCCGGCAACAATTAATAGACTGG \\
\hline BLF2-DKS-Calm1-rev & GCTGATCTGCAGATTTATCCCAATGCTTAATCAGTGAGGCACCTATCTCAGC \\
\hline Calm1-DKS-BLF2-for & GCATTGGGATAAATCTGCAGATCAGCTTGACCGAAGAACAGATTGC \\
\hline Calm1-GGSGG-rev & CCGCCTCCGGACCCTTTTGCAGTCATCATCTGTACGAATTCTTCATAGTTGACTTGTCC \\
\hline BLF1-GGSGG-Calm1-for & GCAAAAGGGTCCGGAGGCGGTCACCCAGAAACGCTGGTGAAAGTAAAAGATGC \\
\hline BLF2-Calm1-rev & CGGTCAGCTGATCTGCCCAATGCTTAATCAGTGAGGCACCTATCTCAGC \\
\hline Calm1-BLF2-for & GCATTGGGCAGATCAGCTGACCGAAGAACAGATTGC \\
\hline Calm1-BLF1-rev & CGTTTCTGGGTGTTTTGCAGTCATCATCTGTACGAATTCTTCATAGTTGACTTGTCC \\
\hline BLF1-Calm1-for & CGTACAGATGATGACTGCAAAACACCCAGAAACGCTGGTGAAAGTAAAAGATGC \\
\hline BLF1-rev & CCACGCACTAGTCTATTAGCCAGTTAATAGTTTGCGCAACGTTGTTGC \\
\hline \multicolumn{2}{|l|}{ P5 and P6 Primers } \\
\hline Bla24(BamHI)-for & CGG TGG GAT CC 7 \\
\hline BLA226-DKS-Calm1-rev & GCTGATCTGCAGATTTATCAGCCGGAAGGGCCGAGC \\
\hline Calm1-DKS-BLA226-for & CCGGCTGATAAATCTGCAGATCAGCTGACCGAAGAACAGATTGC \\
\hline Calm1-GGSGG-rev & CCGCCTCCGGACCCTTTTGCAGTCATCATCTGTACGAATTCTTCATAGTTGACTTGTCC \\
\hline BLA227-link-Calm1-for & GCAAAAGGGTCCGGAGGCGGTGGCTGGTTTATTGCTGATAAATCTGGAGCC \\
\hline BLA226-Calm1-rev & GCTGATCTGCAGAAGCCGGAAGGGCCGAGC \\
\hline Calm1 -BLA226-for & CCTTCCGGCTTCTGCAGATCAGCTGACCGAAGAACAGATTGC \\
\hline Calm1-BLA227-rev & GCCTCCGGATTTTGCAGTCATCATCTGTACGAATTCTTCATAGTTGACTTGTCC \\
\hline BLA227-Calm1-for & CGTACAGATGATGACTGCAAAATCCGGAGGCTGGTTTATTGCTGATAAATCTGGAGC \\
\hline BLA(Spel)-rev & CCACGAAGCTTACTAGTCTATTACCAATGCTTAATCAGTGAGGCACCTATCTCAGC \\
\hline \multicolumn{2}{|l|}{ P7 and P8 primers } \\
\hline Bla24(BamHI)-for & CGG TGG GAT CC TAT GCA CCC AGA AAC GCT GGT GAA AGT AAA AGA TGC \\
\hline BLA170-DKS-Calm1-rev & GGTCAGCTGATCTGCAGATTTATCGGCTTCATTCAGCTCCGGTTCC \\
\hline Calm1-DKS-BLA170-for & CCGATAAATCTGCAGATCAGCTGACCGAAGAACAGATTGC \\
\hline Calm1-GGSGG-rev & CCGCCTCCGGACCCTTTTGCAGTCATCATCTGTACGAATTCTTCATAGTTGACTTGTCC \\
\hline BLA170-GSGGG-Calm1-for & GCAAAAGGGTCCGGAGGCGGTGCCATACCAAACGACGAGCG \\
\hline BLA170-Calm1-rev & GCTGATCTGCAGAGGCTTCATTCAGCTCCGGTTCCC \\
\hline Calm1-BLA170-for & GCTGAATGAAGCCTCCTGCAGATCAGCTGACCGAAGAACAGATTGC \\
\hline Calm1-BLA170-rev & GCTCCGGATTTTGCAGTCATCATCTGTACGAATTCTTCATAGTTGACTTGTCC \\
\hline BLA170-Calm1-for & CGTACAGATGATGACTGCAAAATCCGGAGCCATACCAAACGACGAGCGTGACACC \\
\hline & CCACGAAGCTTACTAGTCTATTACCAATGCTTAATCAGTGAGGCACCTATCTCAGC \\
\hline \multicolumn{2}{|l|}{$\begin{array}{l}\text { Control and Expression } \\
\text { Primers }\end{array}$} \\
\hline Calm1(BamHI)-for & GAGGAAGGATCCATGGCAGATCAGCTGACCGAA \\
\hline Calm1(Spel)-rev & CGACTAACTAGTCTATCATTTTTGCAGTCATCATCTGTACGAATTCTTCATAGTTGAC \\
\hline CaMopt-for & CTGTCAGGATCCATATGGCAGATCAGCTGACCGAAGAACAG \\
\hline CaMopt(Xhol)-rev & ACGTCACTCGAGTTTTGCAGTCATCATCTGTACGAATTCTTCATAG \\
\hline BLA2opt-for & CTGTCAGGATCCATATGCTGCTTACTCTGGCTTCCCGC \\
\hline BLA1opt(Xhol)-rev & ACGTCACTCGAGGCCAGTTAACAGTTTGCGCAACG \\
\hline
\end{tabular}




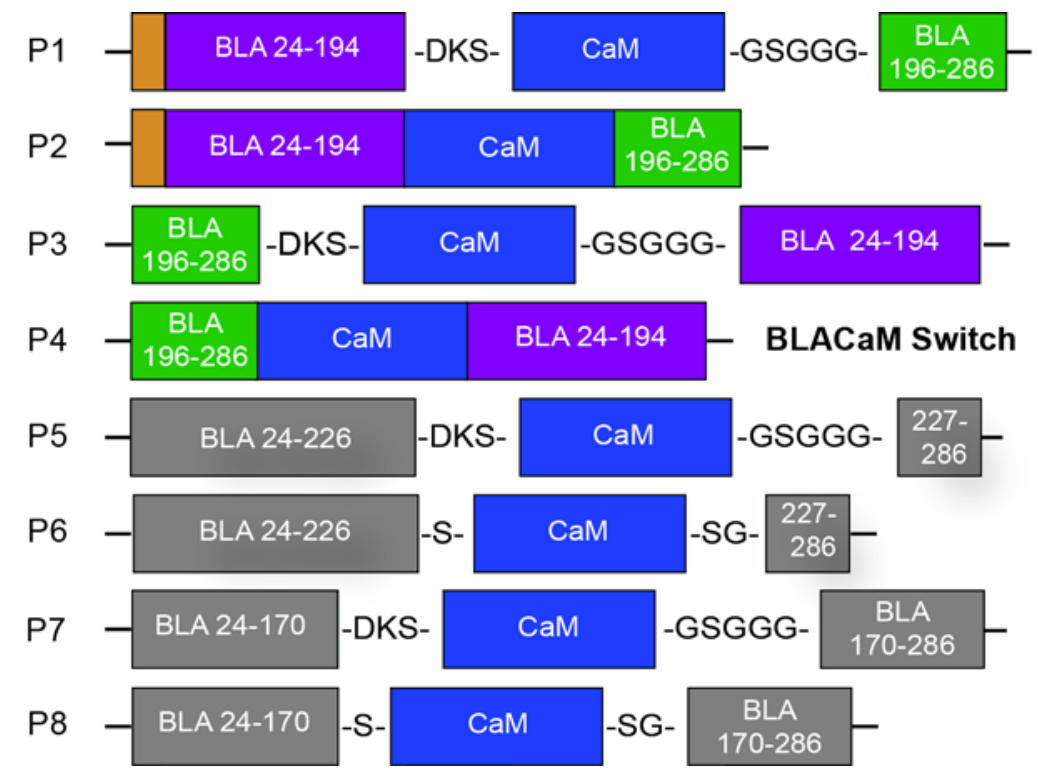

Figure S1. Schematic of $\beta$-lactamase-calmodulin fusions. Calmodulin (blue) fused to either PCA fragments BLF1 (purple) and BLF2 (green), or other BLA fragments (grey). P1 and P2 also contain the wt-BLA periplasmic export sequence (orange). 


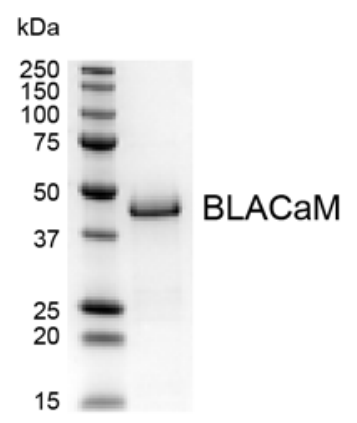

Figure S2. SDS-PAGE confirming the purity of BLACaM after codon optimization, expression in E. coli, and affinity purification. 
a

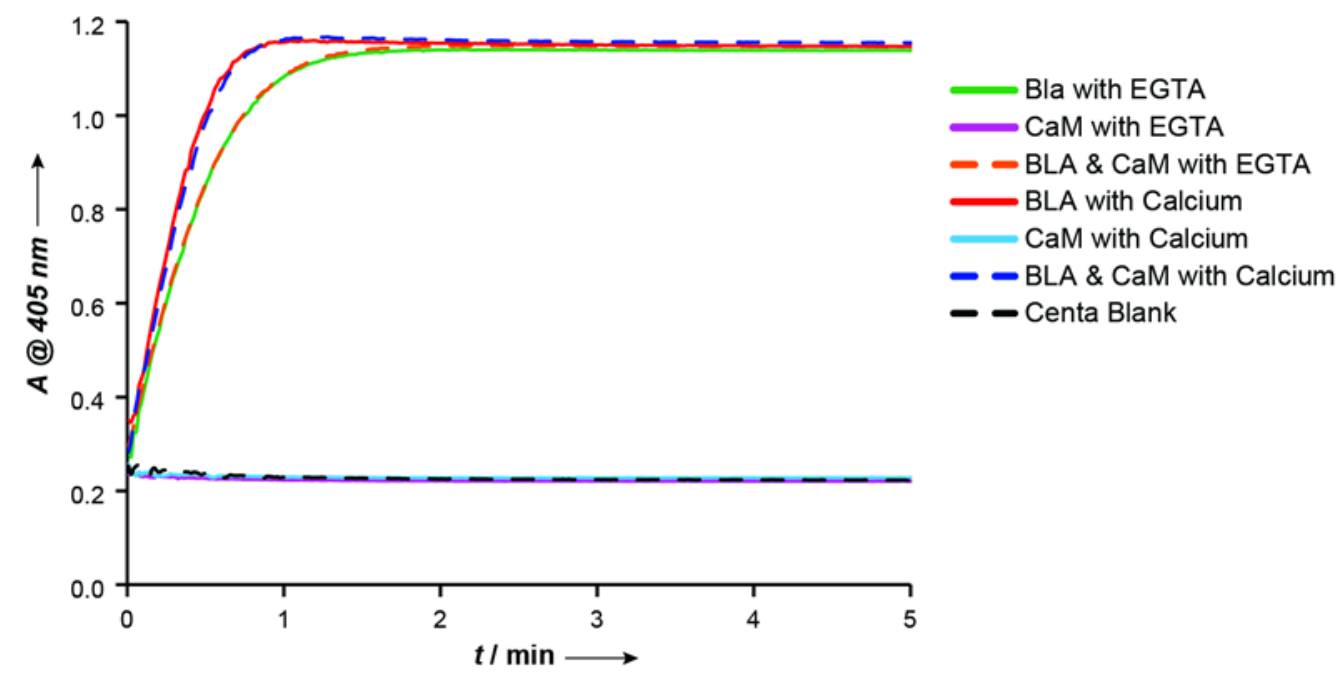

b

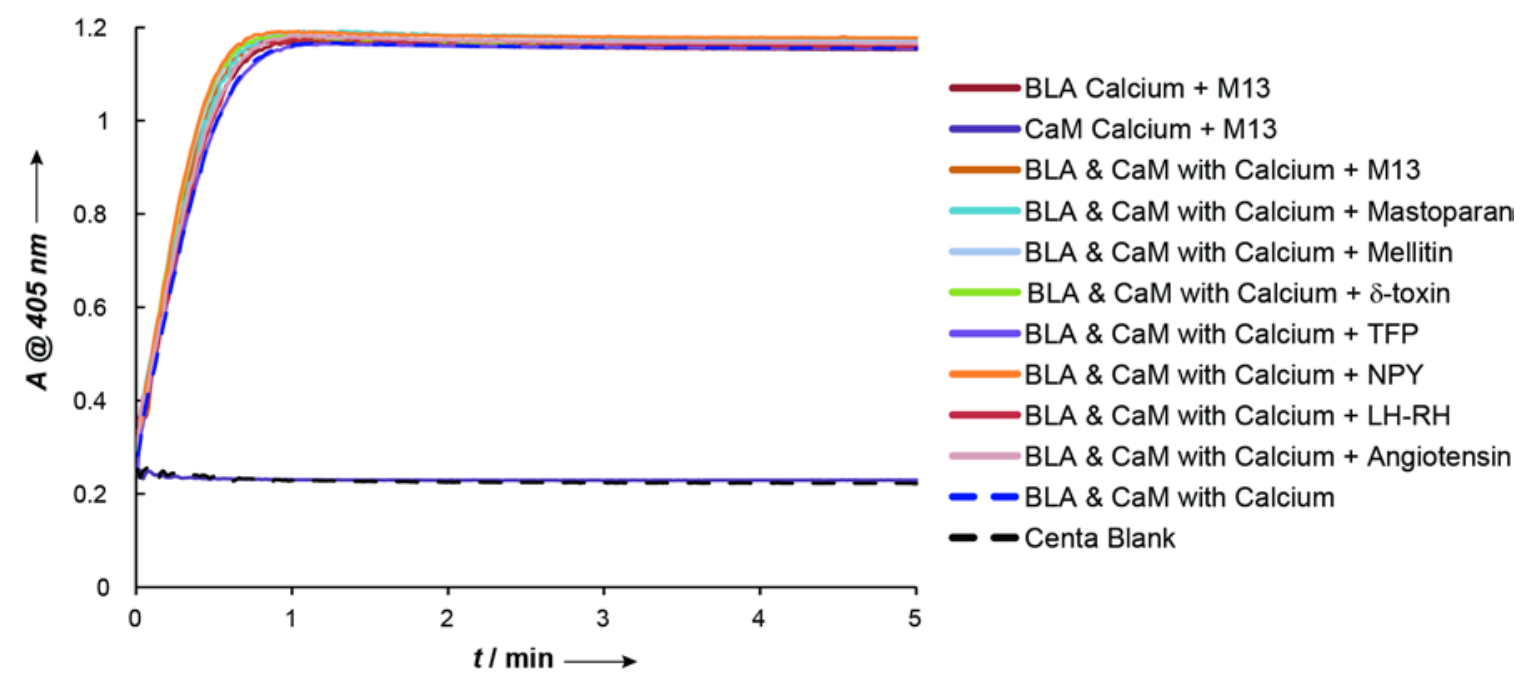

Figure S3. Buffer condition effects on CENTA ${ }^{\mathrm{TM}}$ hydrolysis activity of wt-BLA and wt-CaM proteins. Substrate hydrolysis by BLA, CaM, and BLA and CaM combined is monitored as absorbance at $405 \mathrm{~nm}$ in conditions such as (a) EGTA or calcium only and (b) calcium and the peptides M13 (smMLCK), mastoparan, mellitin, $\delta$-toxin, neuropeptide $Y$ (NPY), luteinizinghormone releasing-hormone (LH-RH), angiotensin, and small molecule trifluoperazine (TFP). 
a

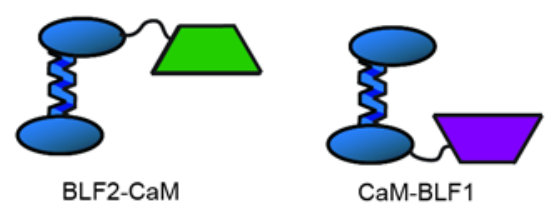

b

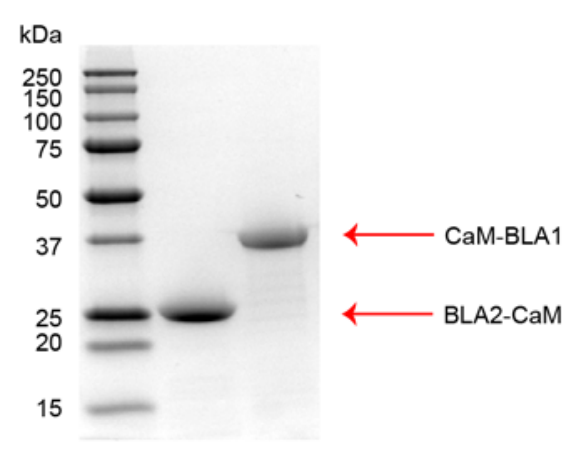

C

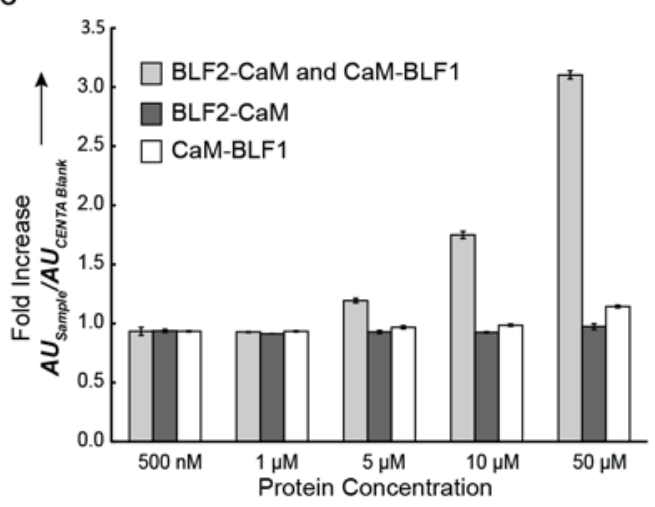

Figure S4. Results from control experiments to monitor the propensity for BLACaM fragments to spontaneously assemble in an intermolecular fashion and reconstitute an active enzyme. a) Schematic depicting the BLACaM fragments BLF1-CaM and CaM-BLF21. b) SDS page gel of the purified fragments after expression in E. coli and affinity purification. c) Total substrate hydrolysis was monitored in HEPES buffer for each of the fragments individually and combined at varying equimolar concentrations. Sample absorbances were normalized to blank samples of the assay substrate in the absence of any enzyme. 


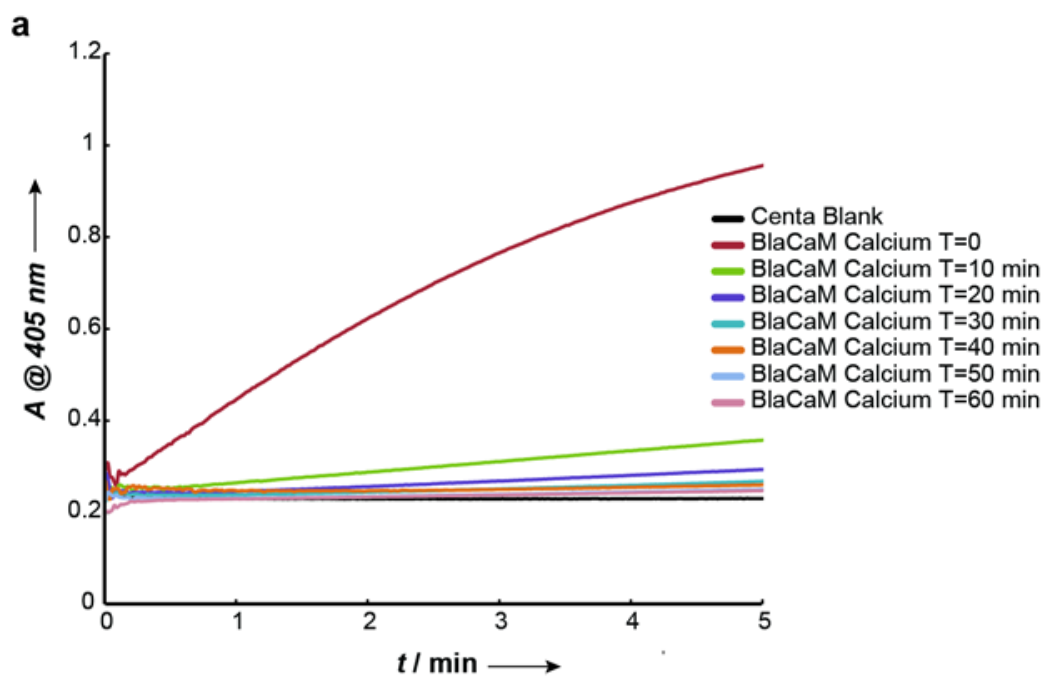

b

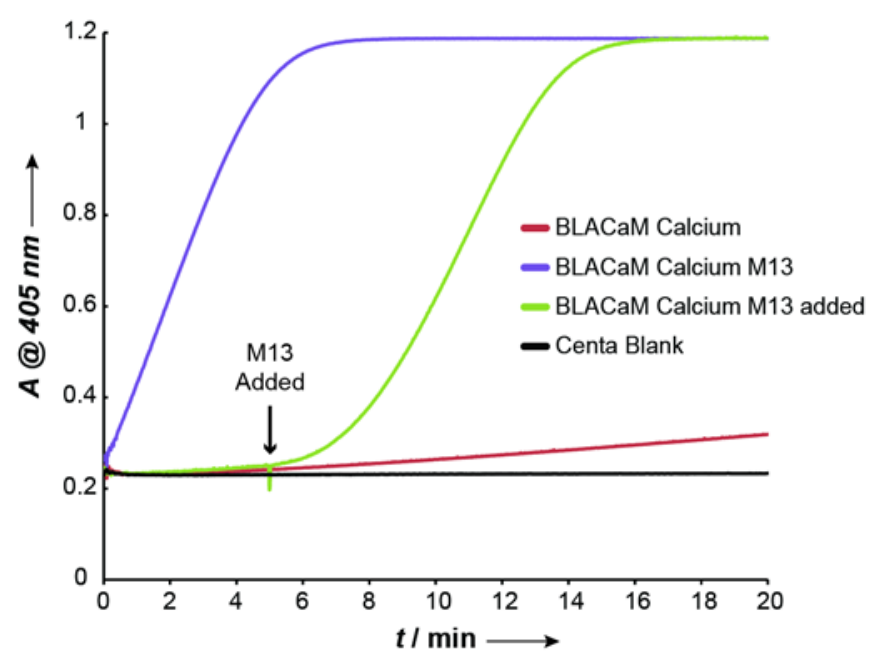

Figure S5. Optimization of activity assay conditions for BLACaM. a) Hydrolysis of CENTA ${ }^{\text {TM }}$ substrate by BLACaM was monitored directly after the purification procedure after incubation for different lengths of time in the presence of $5 \mathrm{mM}$ calcium. b) Comparison of BLACaM activity after preincubation for 60 min with calcium alone (red), or calcium plus M13 (purple). In order to monitor enzyme activation in real time, the assay was also performed with a preincubation for 60 min in calcium alone and addition of M13 to the assay solution 5 min after the start of the assay (green). 
a

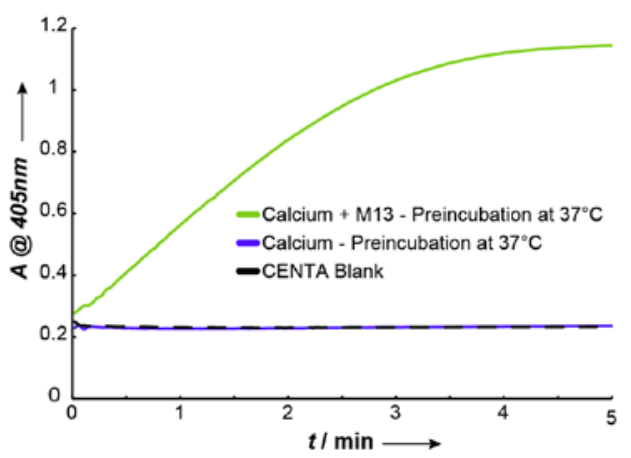

C

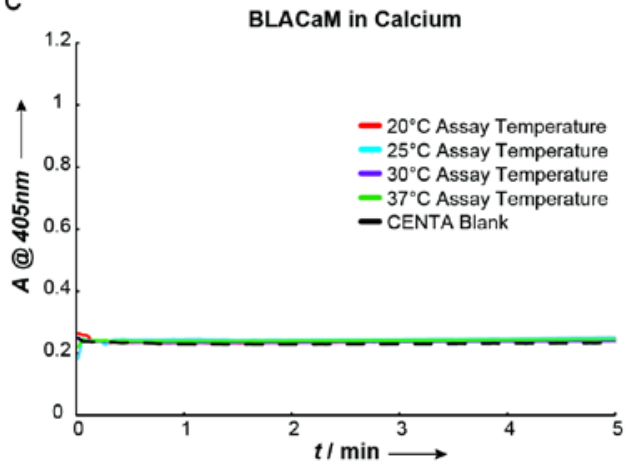

b

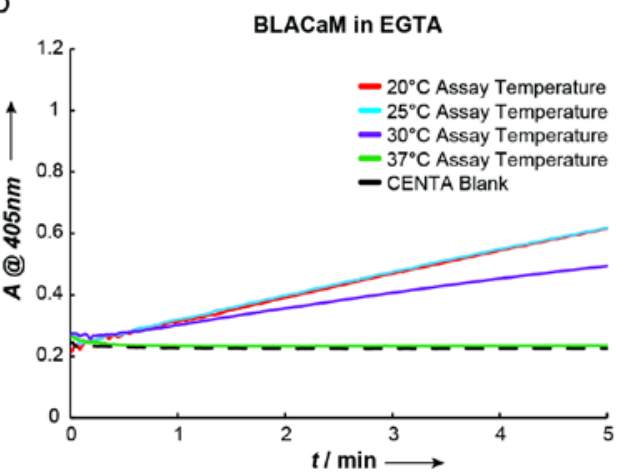

d

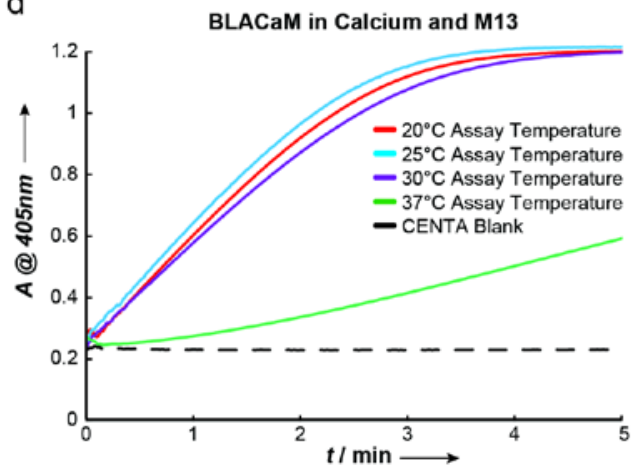

Figure S6. Effects of temperature on BLACaM activity. a) BLACaM with either $5 \mathrm{mM}$ calcium (purple) or $5 \mathrm{mM}$ calcium and M13 (green) was preincubated at $37^{\circ} \mathrm{C}$ for $10 \mathrm{~min}$ in $5 \mathrm{mM} \mathrm{Ca}^{2+}$, then equilibrated at $25^{\circ} \mathrm{C}$ before CENTA ${ }^{\mathrm{TM}}$ assays were performed at $25^{\circ} \mathrm{C}$. Alternatively BLACaM was preincubated with calcium for 50 minutes at room temperature, followed by a 10 min equilibration at either $20,25,30$ or $37^{\circ} \mathrm{C}$ before assays were performed at the same temperatures. Assays were preformed in the presence of 1 mM EGTA (b), 5 mM calcium (c), or $5 \mathrm{mM}$ calcium plus $500 \mathrm{nM}$ M13 peptide (d). 